\title{
Some New Iterative Methods for Nonlinear Equations
}

\author{
Muhammad Aslam Noor, ${ }^{1,2}$ Khalida Inayat Noor, $^{1}$ Eisa Al-Said, ${ }^{2}$ \\ and Muhammad Waseem ${ }^{1}$ \\ ${ }^{1}$ Department of Mathematics, COMSATS Institute of Information Technology, Park Road, \\ Islamabad 44000, Pakistan \\ ${ }^{2}$ Mathematics Department, College of Science, King Saud University, Riyadh 11451, Saudi Arabia
}

Correspondence should be addressed to Muhammad Aslam Noor, mnoor.c@ksu.edu.sa

Received 1 October 2010; Accepted 20 December 2010

Academic Editor: Carlo Cattani

Copyright (C) 2010 Muhammad Aslam Noor et al. This is an open access article distributed under the Creative Commons Attribution License, which permits unrestricted use, distribution, and reproduction in any medium, provided the original work is properly cited.

We suggest and analyze some new iterative methods for solving the nonlinear equations $f(x)=0$ using the decomposition technique coupled with the system of equations. We prove that new methods have convergence of fourth order. Several numerical examples are given to illustrate the efficiency and performance of the new methods. Comparison with other similar methods is given.

\section{Introduction}

It is well known that a wide class of problem which arises in several branches of pure and applied science can be studied in the general framework of the nonlinear equations $f(x)=0$. Due to their importance, several numerical methods have been suggested and analyzed under certain conditions. These numerical methods have been constructed using different techniques such as Taylor series, homotopy perturbation method and its variant forms, quadrature formula, variational iteration method, and decomposition method; see, for example, [1-19]. To implement the decomposition method, one has to calculate the so-called Adomian polynomial, which is itself a difficult problem. Other technique have also their limitations. To overcome these difficulties, several other techniques have been suggested and analyzed for solving the nonlinear equations. One of the decompositions is due to DaftardarGejji and Jafari [6]. In this paper, we use this decomposition method to construct some new iterative methods. To apply this technique, we first use the new series representation of the nonlinear function, which is obtained by using the quadrature formula and the fundamental theorem of calculus. We rewrite the nonlinear equation as a coupled system of nonlinear 
equations. Applying the decomposition of Daftardar-Gejji and Jafari [6], we are able to construct some new iterative methods for solving the nonlinear equations. Our method of construction of these iterative methods is very simple as compared with other methods. We also prove convergence of the proposed methods, which is of order four. Several numerical examples are given to illustrate the efficiency and the performance of the new iterative methods. Our results can be considered as an important improvement and refinement of the previously results.

\section{Iterative Methods}

Consider the nonlinear equation

$$
f(x)=0 .
$$

Using the quadrature formula and the fundamental theorem of calculus, (2.1) can be written as

$$
f(x)=f(\gamma)+(x-\gamma)\left[\frac{f^{\prime}(\gamma)+2 f^{\prime}((\gamma+x) / 2)+f^{\prime}(x)}{4}\right]=0,
$$

where $\gamma$ is an initial guess sufficiently close to $\alpha$, which is a simple root of (2.1). We can rewrite the nonlinear equation (2.1) as a coupled system

$$
\begin{gathered}
f(\gamma)+(x-\gamma)\left[\frac{f^{\prime}(\gamma)+2 f^{\prime}((\gamma+x) / 2)+f^{\prime}(x)}{4}\right]+g(x)=0, \\
g(x)=f(x)-f(\gamma)-(x-\gamma)\left[\frac{f^{\prime}(\gamma)+2 f^{\prime}((\gamma+x) / 2)+f^{\prime}(x)}{4}\right] .
\end{gathered}
$$

From (2.3), we have

$$
x=\gamma-4\left[\frac{f(\gamma)+g(x)}{f^{\prime}(\gamma)+2 f^{\prime}((\gamma+x) / 2)+f^{\prime}(x)}\right]=c+N(x),
$$

where

$$
\begin{gathered}
c=\gamma, \\
N(x)=-4\left[\frac{f(\gamma)+g(x)}{f^{\prime}(\gamma)+2 f^{\prime}((\gamma+x) / 2)+f^{\prime}(x)}\right] .
\end{gathered}
$$

It is clear that the operator $N(x)$ is nonlinear. We now construct a sequence of higher-order iterative methods by using the decomposition technique, which is mainly due to DaftardarGejji and Jafari [6]. This decomposition of the nonlinear function $N(x)$ is quite different from 
that of Adomian decomposition. In this method, one does not have to calculate the so-called the Adomian polynomial, which is another advantage of this decomposition. The main idea of this technique is to look for a solution having the series form

$$
x=\sum_{i=0}^{\infty} x_{i}
$$

The nonlinear operator $N$ can be decomposed as

$$
N(x)=N\left(x_{0}\right)+\sum_{i=1}^{\infty}\left\{N\left(\sum_{j=0}^{i} x_{j}\right)-N\left(\sum_{j=0}^{i-1} x_{j}\right)\right\} .
$$

Combining (2.5), (2.8), and (2.9), we have

$$
\sum_{i=0}^{\infty} x_{i}=c+N\left(x_{0}\right)+\sum_{i=1}^{\infty}\left\{N\left(\sum_{j=0}^{i} x_{j}\right)-N\left(\sum_{j=0}^{i-1} x_{j}\right)\right\} .
$$

Thus, we have the following iterative scheme:

$$
\begin{aligned}
x_{0} & =c, \\
x_{1} & =N\left(x_{0}\right), \\
x_{2} & =N\left(x_{0}+x_{1}\right)-N\left(x_{0}\right), \\
\vdots & \\
x_{m+1} & =N\left(\sum_{j=0}^{m} x_{j}\right)-N\left(\sum_{j=0}^{m-1} x_{j}\right), \quad m=1,2, \ldots
\end{aligned}
$$

Then,

$$
\begin{gathered}
x_{1}+x_{2}+\cdots+x_{m+1}=N\left(x_{0}+x_{1}+\cdots+x_{m}\right), \quad m=1,2, \ldots, \\
x=c+\sum_{i=1}^{\infty} x_{i} .
\end{gathered}
$$

It can be shown that the series $\sum_{i=0}^{\infty} x_{i}$ converges absolutely and uniformly to a unique solution of (2.6). see [6].

From (2.7) and (2.12), we have

$$
x_{0}=c=\gamma .
$$


From (2.4), (2.8) and using the idea of Yun [19], we obtain

$$
\begin{array}{r}
g\left(x_{0}\right)=0, \\
x_{1}=N\left(x_{0}\right)=-4\left[\frac{f(\gamma)+g\left(x_{0}\right)}{f^{\prime}(\gamma)+2 f^{\prime}\left(\left(\gamma+x_{0}\right) / 2\right)+f^{\prime}\left(x_{0}\right)}\right]=-4\left[\frac{f(\gamma)}{f^{\prime}(\gamma)+2 f^{\prime}\left(\left(\gamma+x_{0}\right) / 2\right)+f^{\prime}\left(x_{0}\right)}\right] .
\end{array}
$$

Note that $x$ is approximated by

$$
X_{m}=x_{0}+x_{1}+x_{2}+\cdots+x_{m}
$$

where $\lim _{m \rightarrow \infty} X_{m}=x$.

For $m=0$,

$$
x \approx X_{0}=x_{0}=c=\gamma .
$$

For $m=1$,

$$
x \approx X_{1}=x_{0}+x_{1}=\gamma-4\left[\frac{f(\gamma)}{f^{\prime}(\gamma)+2 f^{\prime}\left(\left(\gamma+x_{0}\right) / 2\right)+f^{\prime}\left(x_{0}\right)}\right]=x_{0}-\frac{f\left(x_{0}\right)}{f^{\prime}\left(x_{0}\right)} .
$$

This formulation allows us to suggest the following one-step iterative method for solving the nonlinear equation (2.1).

Algorithm 2.1. For a given $x_{0}$, compute the approximate solution $x_{n+1}$ by the following iterative scheme:

$$
x_{n+1}=x_{n}-\frac{f\left(x_{n}\right)}{f^{\prime}\left(x_{n}\right)}, \quad f^{\prime}\left(x_{n}\right) \neq 0, \quad n=0,1,2, \ldots
$$

It is a well-known Newton method for solving nonlinear equations (2.1), which has secondorder convergence.

From (2.1), we have

$$
x_{0}+x_{1}-\gamma=-\frac{f(\gamma)}{f^{\prime}(\gamma)} .
$$


From (2.4), (2.8) and using the idea of Yun [19], we have

$$
\begin{aligned}
g\left(x_{0}+x_{1}\right) & =f\left(x_{0}+x_{1}\right)-f(\gamma)-\left(x_{0}+x_{1}-\gamma\right)\left[\frac{f^{\prime}(\gamma)+2 f^{\prime}\left(\left(\gamma+x_{0}+x_{1}\right) / 2\right)+f^{\prime}\left(x_{0}+x_{1}\right)}{4}\right] \\
& =f\left(x_{0}+x_{1}\right)-f(\gamma)+\frac{f(\gamma)}{4 f^{\prime}(\gamma)}\left[f^{\prime}(\gamma)+2 f^{\prime}\left(\frac{\gamma+x_{0}+x_{1}}{2}\right)+f^{\prime}\left(x_{0}+x_{1}\right)\right], \\
x_{1}+x_{2} & =N\left(x_{0}+x_{1}\right)=-4\left[\frac{f(\gamma)+g\left(x_{0}+x_{1}\right)}{f^{\prime}(\gamma)+2 f^{\prime}\left(\left(\gamma+x_{0}+x_{1}\right) / 2\right)+f^{\prime}\left(x_{0}+x_{1}\right)}\right] \\
& =-\frac{f(\gamma)}{f^{\prime}(\gamma)}-\frac{4 f\left(x_{0}+x_{1}\right)}{f^{\prime}(\gamma)+2 f^{\prime}\left(\left(\gamma+x_{0}+x_{1}\right) / 2\right)+f^{\prime}\left(x_{0}+x_{1}\right)} .
\end{aligned}
$$

For $m=2$,

$$
\begin{aligned}
x \approx X_{2} & =x_{0}+x_{1}+x_{2}=c+N\left(x_{0}+x_{1}\right) \\
& =\gamma-\frac{f(\gamma)}{f^{\prime}(\gamma)}-\frac{4 f\left(x_{0}+x_{1}\right)}{f^{\prime}(\gamma)+2 f^{\prime}\left(\left(\gamma+x_{0}+x_{1}\right) / 2\right)+f^{\prime}\left(x_{0}+x_{1}\right)} .
\end{aligned}
$$

Using this relation, we can suggest the following two-step iterative method for solving nonlinear equation (2.1).

Algorithm 2.2. For a given $x_{0}$, compute the approximate solution $x_{n+1}$ by the iterative following scheme:

$$
\begin{gathered}
y_{n}=x_{n}-\frac{f\left(x_{n}\right)}{f^{\prime}\left(x_{n}\right)}, \\
x_{n+1}=y_{n}-\frac{4 f\left(y_{n}\right)}{f^{\prime}\left(x_{n}\right)+2 f^{\prime}\left(\left(x_{n}+y_{n}\right) / 2\right)+f^{\prime}\left(y_{n}\right)}, \quad n=0,1,2, \ldots
\end{gathered}
$$

From (2.22), we obtain

$$
x_{0}+x_{1}+x_{2}-\gamma=-\frac{f(\gamma)}{f^{\prime}(\gamma)}-\frac{4 f\left(x_{0}+x_{1}\right)}{f^{\prime}(\gamma)+2 f^{\prime}\left(\left(\gamma+x_{0}+x_{1}\right) / 2\right)+f^{\prime}\left(x_{0}+x_{1}\right)} .
$$


From (2.4), (2.8) and using the idea of Yun [19], we get

$$
\begin{aligned}
g\left(x_{0}+\right. & \left.x_{1}+x_{2}\right) \\
= & f\left(x_{0}+x_{1}+x_{2}\right)-f(\gamma)-\left(x_{0}+x_{1}+x_{2}-\gamma\right) \\
& \times\left[\frac{f^{\prime}(\gamma)+2 f^{\prime}\left(\left(\gamma+x_{0}+x_{1}+x_{2}\right) / 2\right)+f^{\prime}\left(x_{0}+x_{1}+x_{2}\right)}{4}\right] \\
= & f\left(x_{0}+x_{1}+x_{2}\right)-f(\gamma) \\
& -\frac{1}{4}\left(-\frac{f(\gamma)}{f^{\prime}(\gamma)}-\frac{4 f\left(x_{0}+x_{1}\right)}{f^{\prime}(\gamma)+2 f^{\prime}\left(\left(\gamma+x_{0}+x_{1}\right) / 2\right)+f^{\prime}\left(x_{0}+x_{1}\right)}\right) \\
& \times\left[f^{\prime}(\gamma)+2 f^{\prime}\left(\frac{\gamma+x_{0}+x_{1}+x_{2}}{2}\right)+f^{\prime}\left(x_{0}+x_{1}+x_{2}\right)\right],
\end{aligned}
$$

$x_{1}+x_{2}+x_{3}$

$$
\begin{aligned}
= & N\left(x_{0}+x_{1}+x_{2}\right)=-4\left[\frac{f(\gamma)+g\left(x_{0}+x_{1}+x_{2}\right)}{f^{\prime}(\gamma)+2 f^{\prime}\left(\left(\gamma+x_{0}+x_{1}+x_{2}\right) / 2\right)+f^{\prime}\left(x_{0}+x_{1}+x_{2}\right)}\right] \\
= & -\frac{f(\gamma)}{f^{\prime}(\gamma)}-\frac{4 f\left(x_{0}+x_{1}\right)}{f^{\prime}(\gamma)+2 f^{\prime}\left(\left(\gamma+x_{0}+x_{1}\right) / 2\right)+f^{\prime}\left(x_{0}+x_{1}\right)} \\
& -\frac{4 f\left(x_{0}+x_{1}+x_{2}\right)}{f^{\prime}(\gamma)+2 f^{\prime}\left(\left(\gamma+x_{0}+x_{1}+x_{2}\right) / 2\right)+f^{\prime}\left(x_{0}+x_{1}+x_{2}\right)} .
\end{aligned}
$$

For $m=3$,

$$
\begin{aligned}
x \approx X_{3}= & x_{0}+x_{1}+x_{2}+x_{3}=c+N\left(x_{0}+x_{1}+x_{2}\right) \\
= & \gamma-\frac{f(\gamma)}{f^{\prime}(\gamma)}-\frac{4 f\left(x_{0}+x_{1}\right)}{f^{\prime}(\gamma)+2 f^{\prime}\left(\left(\gamma+x_{0}+x_{1}\right) / 2\right)+f^{\prime}\left(x_{0}+x_{1}\right)} \\
& -\frac{4 f\left(x_{0}+x_{1}+x_{2}\right)}{f^{\prime}(\gamma)+2 f^{\prime}\left(\left(\gamma+x_{0}+x_{1}+x_{2}\right) / 2\right)+f^{\prime}\left(x_{0}+x_{1}+x_{2}\right)} .
\end{aligned}
$$

Using this formulation, we can suggest the following three-step iterative method for solving nonlinear equation (2.1). 
Algorithm 2.3. For a given $x_{0}$, compute the approximate solution $x_{n+1}$ by the iterative following scheme.

$$
\begin{gathered}
y_{n}=x_{n}-\frac{f\left(x_{n}\right)}{f^{\prime}\left(x_{n}\right)}, \\
z_{n}=y_{n}-\frac{4 f\left(y_{n}\right)}{f^{\prime}\left(x_{n}\right)+2 f^{\prime}\left(\left(x_{n}+y_{n}\right) / 2\right)+f^{\prime}\left(y_{n}\right)}, \\
x_{n+1}=z_{n}-\frac{4 f\left(z_{n}\right)}{f^{\prime}\left(x_{n}\right)+2 f^{\prime}\left(\left(x_{n}+z_{n}\right) / 2\right)+f^{\prime}\left(z_{n}\right)}, \quad n=0,1,2, \ldots
\end{gathered}
$$

\section{Convergence Analysis}

In this section, we consider the convergence criteria of the iterative methods developed in Section 2. In a similar way, one can consider the convergence of other algorithms.

Theorem 3.1. Let $\alpha \in I$ be a simple zero of sufficiently differentiable function $f: I \subseteq R \rightarrow R$ for an open interval I. If $x_{0}$ is sufficiently close to $\alpha$, then the iterative methods defined by Algorithm 2.3 has fourth-order convergence.

Proof. Let $\alpha$ be a simple zero of $f$. Then, by expanding $f\left(x_{n}\right)$ and $f^{\prime}\left(x_{n}\right)$ in Taylor's Series about $\alpha$, we have

$$
\begin{gathered}
f\left(x_{n}\right)=f^{\prime}(\alpha)\left[e_{n}+c_{2} e_{n}^{2}+c_{3} e_{n}^{3}+c_{4} e_{n}^{4}+O\left(e_{n}^{5}\right)\right], \\
f^{\prime}\left(x_{n}\right)=f^{\prime}(\alpha)\left[1+2 c_{2} e_{n}+3 c_{3} e_{n}^{2}+4 c_{4} e_{n}^{3}+5 c_{5} e_{n}^{4}+O\left(e_{n}^{5}\right)\right],
\end{gathered}
$$

where $c_{k}=(1 / k !)\left(f^{(k)}(\alpha) / f^{\prime}(\alpha)\right), k=2,3, \ldots$ and $e_{n}=x_{n}-\alpha$.

From (3.1) and (3.2), we have

$$
\frac{f\left(x_{n}\right)}{f^{\prime}\left(x_{n}\right)}=e_{n}-c_{2} e_{n}^{2}+2\left(c_{2}^{2}-c_{3}\right) e_{n}^{3}+\left(7 c_{2} c_{3}-4 c_{2}^{3}-3 c_{4}\right) e_{n}^{4}+O\left(e_{n}^{5}\right)
$$

From (3.3), we get

$$
y_{n}=\alpha+c_{2} e_{n}^{2}+2\left(c_{3}-c_{2}^{2}\right) e_{n}^{3}-\left(7 c_{2} c_{3}-4 c_{2}^{3}-3 c_{4}\right) e_{n}^{4}+O\left(e_{n}^{5}\right)
$$


Expanding $f\left(y_{n}\right), f^{\prime}\left(y_{n}\right), f^{\prime}\left(\left(x_{n}+y_{n}\right) / 2\right)$ in Taylor's Series about $\alpha$ and using (3.4), we have

$$
\begin{gathered}
f\left(y_{n}\right)=f^{\prime}(\alpha)\left[c_{2} e_{n}^{2}+\left(-2 c_{2}^{2}+2 c_{3}\right) e_{n}^{3}+\left(5 c_{2}^{3}-7 c_{2} c_{3}+3 c_{4}\right) e_{n}^{4}+O\left(e_{n}^{5}\right)\right], \\
f^{\prime}\left(y_{n}\right)=f^{\prime}(\alpha)\left[1+2 c_{2}^{2} e_{n}^{2}+4\left(c_{2} c_{3}-c_{2}^{3}\right) e_{n}^{3}+\left(-11 c_{2}^{2} c_{3}+6 c_{2} c_{4}+8 c_{2}^{4}\right) e_{n}^{4}+O\left(e_{n}^{5}\right)\right], \\
f^{\prime}\left(\frac{x_{n}+y_{n}}{2}\right)=f^{\prime}(\alpha)\left[1+c_{2} e_{n}+\left(c_{2}^{2}+\frac{3}{4} c_{3}\right) e_{n}^{2}+\left(\frac{7}{2} c_{2} c_{3}+\frac{1}{2} c_{4}-2 c_{2}^{3}\right) e_{n}^{3}\right. \\
\left.+\left(\frac{9}{2} c_{2} c_{4}+4 c_{2}^{4}-\frac{37}{4} c_{2}^{2} c_{3}+3 c_{3}^{2}+\frac{5}{16} c_{5}\right) e_{n}^{4}+O\left(e_{n}^{5}\right)\right] .
\end{gathered}
$$

From (3.2), (3.6), and (3.7), we have

$$
\begin{aligned}
f^{\prime}\left(x_{n}\right)+2 f^{\prime}\left(\frac{x_{n}+y_{n}}{2}\right)+f^{\prime}\left(y_{n}\right) & \\
=f^{\prime}(\alpha)[4 & +4 c_{2} e_{n}+\left(4 c_{2}^{2}+\frac{9}{2} c_{3}\right) e_{n}^{2}+\left(11 c_{2} c_{3}+5 c_{4}-8 c_{2}^{3}\right) e_{n}^{3} \\
& \left.+\left(6 c_{3}^{2}-\frac{59}{2} c_{2}^{2} c_{3}+16 c_{2}^{4}+\frac{45}{8} c_{5}+15 c_{2} c_{4}\right) e_{n}^{4}+O\left(e_{n}^{5}\right)\right] .
\end{aligned}
$$

From (3.5) and (3.8), we obtain

$$
\begin{aligned}
& \frac{4 f\left(y_{n}\right)}{f^{\prime}\left(x_{n}\right)+2 f^{\prime}\left(\left(x_{n}+y_{n}\right) / 2\right)+f^{\prime}\left(y_{n}\right)} \\
& \quad=c_{2} e_{n}^{2}+\left(-3 c_{2}^{2}+2 c_{3}\right) e_{n}^{3}+\left(3 c_{4}+7 c_{2}^{3}-\frac{81}{8} c_{2} c_{3}\right) e_{n}^{4}+O\left(e_{n}^{5}\right) .
\end{aligned}
$$

From (3.4) and (3.9), we have

$$
z_{n}=\alpha+c_{2}^{2} e_{n}^{3}+\left(-3 c_{2}^{3}+\frac{25}{8} c_{2} c_{3}\right) e_{n}^{4}+O\left(e_{n}^{5}\right)
$$

Expanding $f\left(z_{n}\right), f^{\prime}\left(z_{n}\right), f^{\prime}\left(\left(x_{n}+z_{n}\right) / 2\right)$ in Taylor's Series about $\alpha$ and using (3.10), we obtain

$$
\begin{gathered}
f\left(z_{n}\right)=f^{\prime}(\alpha)\left[c_{2}^{2} e_{n}^{3}+\left(-3 c_{2}^{3}+\frac{25}{8} c_{2} c_{3}\right) e_{n}^{4}+O\left(e_{n}^{5}\right)\right] \\
f^{\prime}\left(z_{n}\right)=f^{\prime}(\alpha)\left[1+2 c_{2}^{3} e_{n}^{3}+\left(-6 c_{2}^{4}+\frac{25}{4} c_{2}^{2} c_{3}\right) e_{n}^{4}+O\left(e_{n}^{5}\right)\right] \\
f^{\prime}\left(\frac{x_{n}+z_{n}}{2}\right)=f^{\prime}(\alpha)\left[1+c_{2} e_{n}+\frac{3}{4} c_{3} e_{n}^{2}+\left(\frac{1}{2} c_{4}+c_{2}^{3}\right) e_{n}^{3}+\left(-3 c_{2}^{4}+\frac{37}{8} c_{2}^{2} c_{3}+\frac{5}{16} c_{5}\right) e_{n}^{4}+O\left(e_{n}^{5}\right)\right] .
\end{gathered}
$$


From (3.2), (3.12), and (3.13), we have

$$
\begin{aligned}
f^{\prime}\left(x_{n}\right) & +2 f^{\prime}\left(\frac{x_{n}+z_{n}}{2}\right)+f^{\prime}\left(z_{n}\right) \\
= & f^{\prime}(\alpha)\left[4+4 c_{2} e_{n}+\frac{9}{2} c_{3} e_{n}^{2}+\left(5 c_{4}+4 c_{2}^{3}\right) e_{n}^{3}+\left(-12 c_{2}^{4}+\frac{31}{2} c_{2}^{2} c_{3}+\frac{45}{8} c_{5}\right) e_{n}^{4}+O\left(e_{n}^{5}\right)\right] .
\end{aligned}
$$

From (3.11) and (3.14), we obtain

$$
\frac{4 f\left(z_{n}\right)}{f^{\prime}\left(x_{n}\right)+2 f^{\prime}\left(\left(x_{n}+z_{n}\right) / 2\right)+f^{\prime}\left(z_{n}\right)}=c_{2}^{2} e_{n}^{3}+\left(-4 c_{2}^{3}+\frac{25}{8} c_{2} c_{3}\right) e_{n}^{4}+O\left(e_{n}^{5}\right)
$$

From (3.10) and (3.15), we have

$$
x_{n+1}=\alpha+c_{2}^{3} e_{n}^{4}+O\left(e_{n}^{5}\right)
$$

Thus, we have

$$
e_{n+1}=c_{2}^{3} e_{n}^{4}+O\left(e_{n}^{5}\right)
$$

Error equation (3.17) shows that the Algorithm 2.3 is fourth-order convergent.

\section{Numerical Results}

We now present some examples to illustrate the performance of the newly developed twostep and three-step iterative methods in this paper. We compare Newton method (NM), method of M. A. Noor et al. [9] (NNT), method of Chun [3] (CM), Algorithm 2.2 (NR1), and the Algorithm 2.3 (NR2) introduced in this paper. We used $\varepsilon=10^{-15}$. The following stopping criteria is used for computer programs:
(i) $\left|x_{n+1}-x_{n}\right|<\varepsilon$,
(ii) $\left|f\left(x_{n}\right)\right|<\varepsilon$.

The computational order of convergence $p$ approximated by means of

$$
p \approx \frac{\ln \left(\left|x_{n+1}-x_{n}\right| /\left|x_{n}-x_{n-1}\right|\right)}{\ln \left(\left|x_{n}-x_{n-1}\right| /\left|x_{n-1}-x_{n-2}\right|\right)}
$$




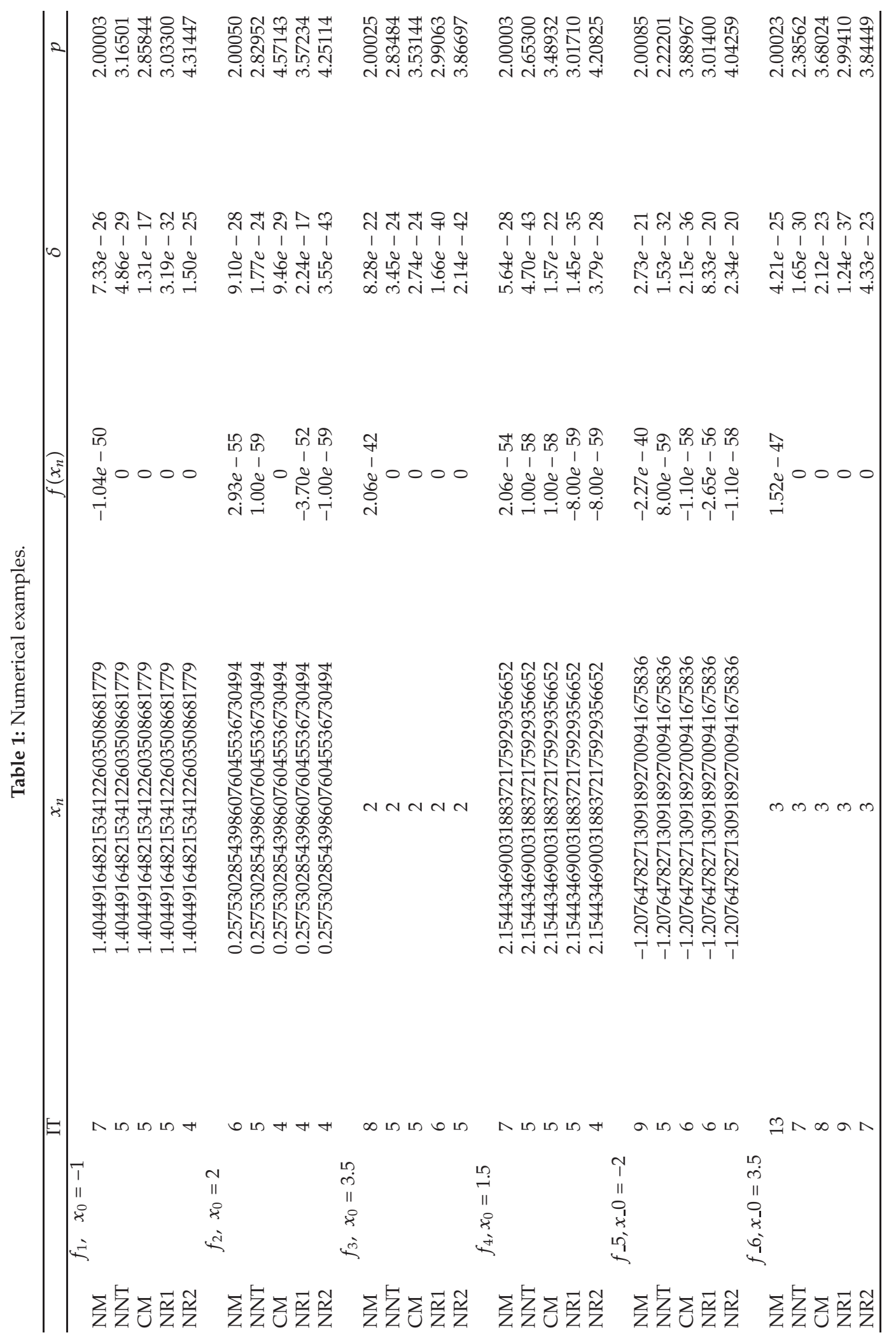


We consider the following nonlinear equations as test problems which are the same as $\mathrm{M}$. Aslam Noor and K. Inayat Noor [10].

$$
\begin{aligned}
& f_{1}(x)=\sin ^{2} x-x^{2}+1, \\
& f_{2}(x)=x^{2}-e^{x}-3 x+2, \\
& f_{3}(x)=(x-1)^{3}-1, \\
& f_{4}(x)=x^{3}-10 \\
& f_{5}(x)=x e^{x^{2}}-\sin ^{2} x+3 \cos x+5, \\
& f_{6}(x)=e^{x^{2}+7 x-30}-1 .
\end{aligned}
$$

\section{Conclusion}

In this paper, we have considered one-step, two-step, and three-step iterative methods for solving nonlinear equations by using a different decomposition technique. Our method of derivation of the iterative methods is very simple as compared with the Adomian decomposition methods. From the Table 1, it is obvious that three-step method introduced in this paper performs better than the fourth-order method of Chun [3]. Using the technique and idea of this paper, one can suggest and analyze higher-order multistep iterative methods for solving nonlinear equations as well as system of nonlinear equations. It is an open problem to extend the technique and ideas of this paper for solving the obstacle problems associated with the variational inequalities and related problems see [20-23] and the references therein. This is another direction for future research.

\section{Acknowledgments}

The authors would like to thank Dr. S. M. Junaid Zaidi, Rector, CIIT, for providing excellent research facilities. This research is supported by the Visiting Professor Program of King Saud University, Riyadh, Saudi Arabia and Research Grant no. KSU.VPP.108.

\section{References}

[1] S. Abbasbandy, "Improving Newton-Raphson method for nonlinear equations by modified Adomian decomposition method," Applied Mathematics and Computation, vol. 145, no. 2-3, pp. 887-893, 2003.

[2] G. Adomian, Nonlinear Stochastic Systems and Applications to Physics, vol. 46 of Mathematics and Its Applications, Kluwer Academic Publishers, Dordrecht, The Netherlands, 1989.

[3] C. Chun, "Iterative methods improving Newton's method by the decomposition method," Computers $\mathcal{E}$ Mathematics with Applications, vol. 50, no. 10-12, pp. 1559-1568, 2005.

[4] C. Chun and Y. Ham, "A one-parameter fourth-order family of iterative methods for nonlinear equations," Applied Mathematics and Computation, vol. 189, no. 1, pp. 610-614, 2007.

[5] C. Chun and Y. Ham, "Some fourth-order modifications of Newton's method," Applied Mathematics and Computation, vol. 197, no. 2, pp. 654-658, 2008.

[6] V. Daftardar-Gejji and H. Jafari, "An iterative method for solving nonlinear functional equations," Journal of Mathematical Analysis and Applications, vol. 316, no. 2, pp. 753-763, 2006.

[7] J.-H. He, "A new iteration method for solving algebraic equations," Applied Mathematics and Computation, vol. 135, no. 1, pp. 81-84, 2003. 
[8] M. Aslam Noor and K. Inayat Noor, "Three-step iterative methods for nonlinear equations," Applied Mathematics and Computation, vol. 183, no. 1, pp. 322-327, 2006.

[9] M. A. Noor, K. I. Noor, S. T. Mohyud-Din, and A. Shabbir, "An iterative method with cubic convergence for nonlinear equations," Applied Mathematics and Computation, vol. 183, no. 2, pp. 1249 1255, 2006.

[10] M. Aslam Noor and K. Inayat Noor, "Some iterative schemes for nonlinear equations," Applied Mathematics and Computation, vol. 183, no. 2, pp. 774-779, 2006.

[11] K. Inayat Noor and M. Aslam Noor, "Predictor-corrector Halley method for nonlinear equations," Applied Mathematics and Computation, vol. 188, no. 2, pp. 1587-1591, 2007.

[12] M. Aslam Noor, K. Inayat Noor, and M. Waseem, "Fourth-order iterative methods for solving nonlinear equations," International Journal of Applied Mathematics and Engineering Sciences, vol. 4, pp. 43-52, 2010.

[13] M. A. Noor, "New iterative schemes for nonlinear equations," Applied Mathematics and Computation, vol. 187, no. 2, pp. 937-943, 2007.

[14] M. A. Noor, "New family of iterative methods for nonlinear equations," Applied Mathematics and Computation, vol. 190, no. 1, pp. 553-558, 2007.

[15] J. M. Ortega and W. C. Rheinboldt, Iterative Solutions of Nonlinear Equations in Several Variables, Academic Press, New York, NY, USA, 1970.

[16] A. M. Ostrowski, Solution of Equations in Euclidean and Banach Space, Academic Press, New York, NY, USA, 1973.

[17] J. F. Traub, Iterative Methods for the Solution of Equations, Prentice-Hall Series in Automatic Computation, Prentice-Hall, Englewood Cliffs, NJ, USA, 1964.

[18] S. Weerakoon and T. G. I. Fernando, “A variant of Newton's method with accelerated third-order convergence," Applied Mathematics Letters, vol. 13, no. 8, pp. 87-93, 2000.

[19] J. H. Yun, "A note on three-step iterative method for nonlinear equations," Applied Mathematics and Computation, vol. 202, no. 1, pp. 401-405, 2008.

[20] M. Aslam Noor, Principles of Variational Inequalities, Lap-Lambert Academic, Saarbrucken, Germany, 2009.

[21] M. Aslam Noor, "Some developments in general variational inequalities," Applied Mathematics and Computation, vol. 152, no. 1, pp. 199-277, 2004.

[22] M. A. Noor, “Extended general variational inequalities," Applied Mathematics Letters, vol. 22, no. 2, pp. 182-186, 2009.

[23] M. A. Noor, K. I. Noor, and T. M. Rassias, "Some aspects of variational inequalities," Journal of Computational and Applied Mathematics, vol. 47, no. 3, pp. 285-312, 1993. 


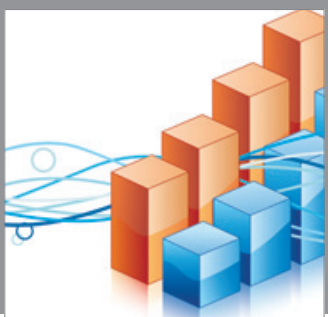

Advances in

Operations Research

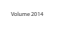

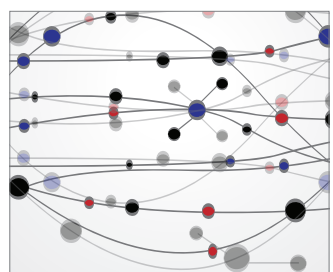

\section{The Scientific} World Journal
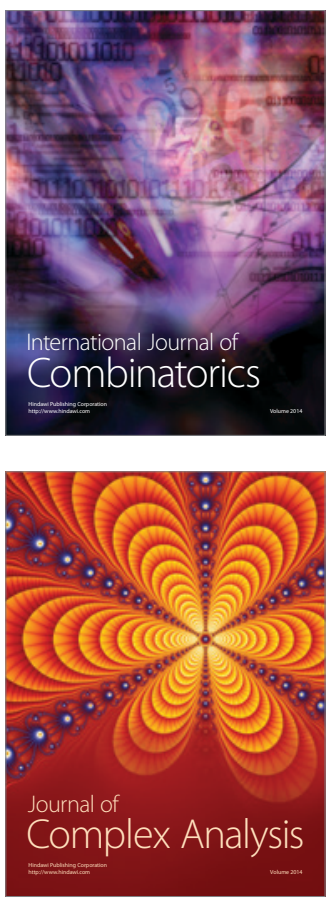

International Journal of

Mathematics and

Mathematical

Sciences
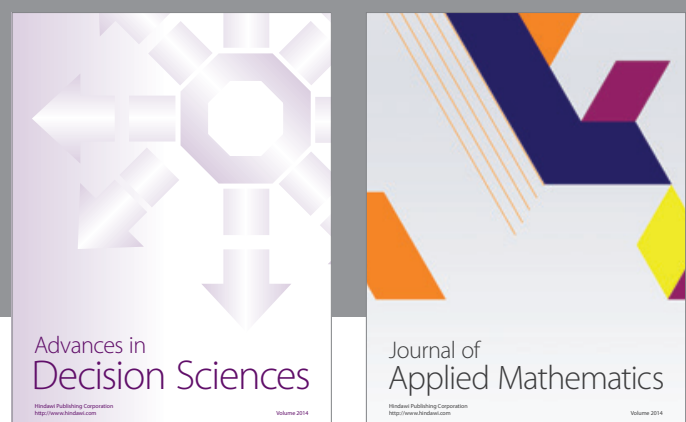

Journal of

Applied Mathematics
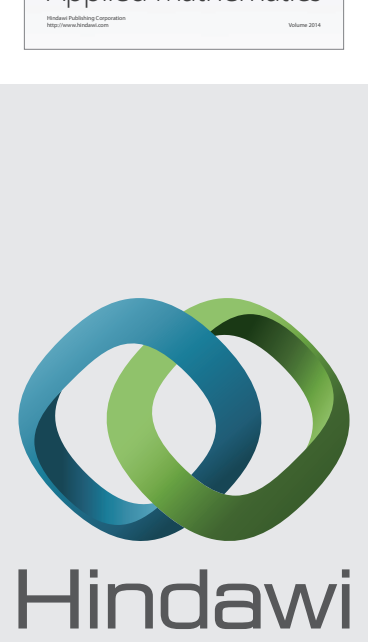

Submit your manuscripts at http://www.hindawi.com
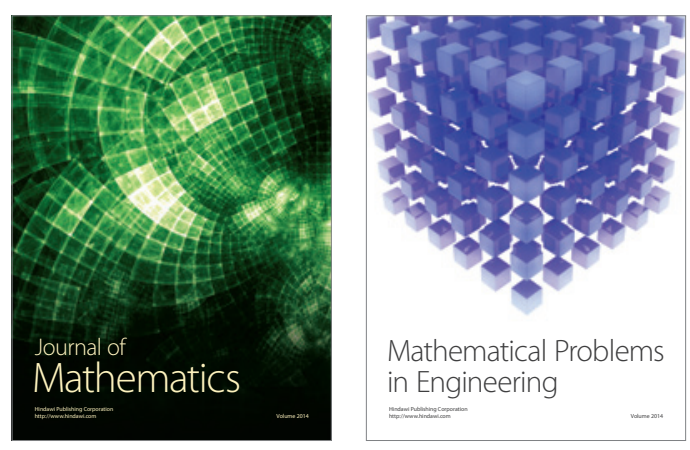

Mathematical Problems in Engineering
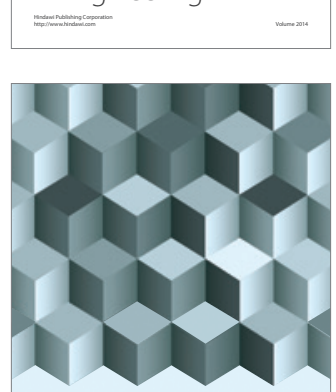

Journal of

Function Spaces
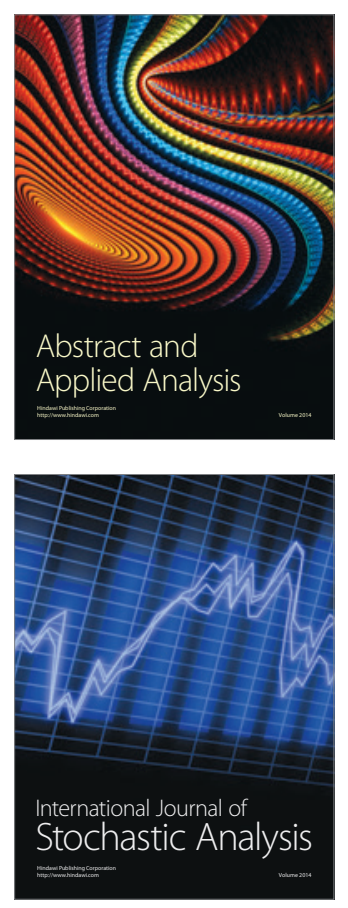

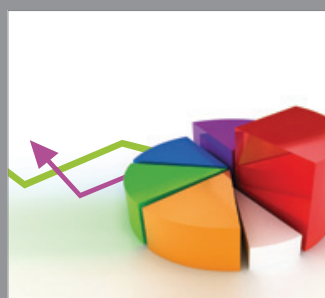

ournal of

Probability and Statistics

Promensencen
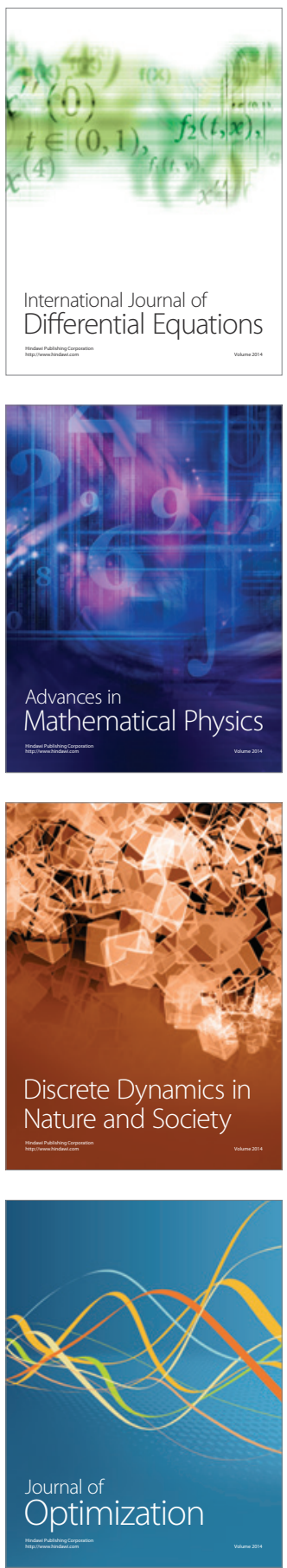